\title{
Tibial acceleration profiles during the menstrual cycle in female athletes.
}

\section{Erik Hohmann}

FRCS, FRCS (Tr\&Orth), MD, PhD

Musculoskeletal Research Unit, Central Queensland University, PO Box 4045, Rockhampton QLD 4700, Australia

ehohmann@optusnet.com.au

\section{Adam L. Bryant L}

BAppSci (Hons), $\mathrm{PhD}$

Centre for Health, Exercise and Sports Medicine Physiotherapy, Melbourne School of Health Sciences, Faculty of Medicine, Dentistry and Health Sciences, The University of Melbourne, Parkville VIC 3010, Australia

albryant@unimelb.edu.au

\section{Elisabeth Livingstone}

MD

Musculoskeletal Research Unit, Central Queensland University, PO Box 4045, Rockhampton QLD 4700, Australia

eli_e@gmx.net

\section{Peter Reaburn 3}

$\mathrm{PhD}$

School of Medical and Applied Sciences, Central Queensland University, Bruce Highway, Rockhampton QLD 4702, Australia

p.reaburn@cqu.edu.au

\section{Kevin Tetsworth}

MD, FRACS

Department of Orthopaedic Surgery, Royal Brisbane Hospital, Herston, Australia

CONROD Professor- Of Orthepaedic Trauma Surgery, Division of Surgery, University of Queensland Medical School, Herston QID 4029, Australia

Kevin_Tetsworth@health.qld.gov.au

\section{Andreas Imhoff}

MD

Department of Orthopaedic Sportsmedicine, University of Technology, Ismaninger Strasse 69, 81675 Munich, Germany

A.Imhoff@1rz.tu-muenchen.de

\section{Corresponding Author:}

Erik Hohmann

Musculoskeletal Research Unit, CQUniversity

PO Box 4045

Rockhampton QLD 4700

Australia

ehohmann@optusnet.com.au

Tel : +61 749206536 


\title{
Tibial acceleration profiles during the menstrual cycle in female athletes.
}

\author{
Abstract \\ Purpose: \\ Fluctuating levels of endogenous estrogen are thought to have an adverse effect on lower \\ limb biomechanics, given the observed higher rate of ACL injury at certain phases of the \\ menstrual cycle. The purpose of this study was to investigate the effects of fluctuating \\ endogenous estrogen levels during the menstrual cycle on acceleration transients at the \\ proximal tibia in young physically active females. \\ Methods:
}

Eleven females aged 16-18 years participated in this study and were compared to a male control group. Female subjects were tested at each of the four phases of the menstrual cycle: menses, follicular, ovulation and luteal. On each test occasion, acceleration transients at the proximal tibia were measured whilst subjects performed an abrupt deceleration task (simulated netball landing).

Results:

No significant differences were found between the different phases of the menstrual cycle for peak tibial acceleration (PTA; $P=0.57$ ), and time to zero tibial acceleration (TZTA; $P=0.59$ ). However, there was a significant difference for time to peak tibial acceleration (TPTA) between menstruation and follicular $(P=0.04)$, menstruation and ovulation $(P=0.001)$, menstruation and luteal phase ( $p=0.002)$, and follicular phase and ovulation $(P=0.007)$. In the male control group, no significant between test session differences were observed for PTA $(P=0.48)$, TZTA $(P=0.08)$ and TPTA $(P=0.29)$. While there were no significant between group differences for PTA $(P=0.21)$ and TZTA $(P=0.48)$, significant between-group differences were observed for TPTA $(P=0.001)$.

Conclusion: 
The results of this project strongly suggest that serum estrogen fluctuations have an effect on tibial acceleration profiles in young female athletes during different phases of the menstrual cycle.

\section{Key Words}

Menstrual cycle, tibial acceleration profiles, anterior cruciate ligament injuries, female athletes 


\section{Introduction}

Female athletes are at significantly higher risk of musculoskeletal injury compared to their male counterparts $[24,35]$, and female athletes participating in the same sport as males are at 2-8 times higher risk for an anterior cruciate ligament (ACL) tear [13,16,48]. Seventy percent of ACL injuries in females occur via non-contact mechanisms $[31,35]$, whereas only $58 \%$ of ACL injuries in males can be classified as non-contact [40]. Numerous factors been implicated for this observed ACL-gender bias, but the cause has not yet been conclusively determined $[17,21,25,37,52]$. However, it appears the higher ACL injury rate in females is most likely multifactorial and related to both intrinsic and extrinsic risk factors [5].

A major intrinsic difference between males and females is their hormonal environment $[12,38,51]$. Specifically, the endogenous estrogen levels of eumenorrheic females fluctuate widely across the four phases (menses, mid-follicular phase, ovulation and mid-luteal phase) of the typical 28-day menstrual cycle. As a result of these hormonal fluctuations, ligament and musculotendinous mechanical properties have been found to vary as a function of menstrual cycle phase $[3,12,40,42]$. Bell at el. showed that changes in hip and knee mechanics were closely correlated with estrogen levels [3]. These changes related to a significanlty greater than expected percentage of anterior cruciate ligament injuries during the ovulatory phase [51]. Oral contraceptive use diminished the significant association between anterior cruciate ligament tear distribution and the ovulatory phase [51].

When performing abrupt deceleration tasks, the lower extremity is subject to considerable impact forces during single-limb ground contact [27,28]. Under these impact loads the lower limb segments experience both compressive forces and acceleration transients $[7,9,18,33]$. Of these, tibial acceleration is one of the main indictors of dynamic knee stability and joint stress 
[33]. For example, McLean et al. have demonstrated a strong relationship between anterior tibial acceleration and ACL strain during a simulated jump landing [32]. Bryant et al. demonstrated that athletes who are able to arrest tibial acceleration faster, tend to display greater knee functionality whether the anterior cruciate ligament is intact, -deficient or reconstructed [7].

To date, no study has investigated whether hormonal fluctuations during the menstrual cycle in female athletes have an effect on the magnitude and temporal characteristics of tibial acceleration profiles during an abrupt deceleration task. The purpose of the present study was to investigate tibial acceleration profiles across the four phases of the menstrual cycle in young, active female athletes. We hypothesized that tibial acceleration profiles would demonstrate higher values of dynamic instability during those phases of the menstrual cycle characterized by higher estrogen levels. 


\section{Material and Methods}

This study uses participants described in our earlier paper [12]. As such, the physiological characteristics of the participants — including their endogenous estrogen levels — have already been reported in full. For this reason, we have abbreviated the relevant parts of the following section to avoid duplication of our earlier work. Ethical clearance was obtained from the Human Ethics Research Review Panel. Participants who agreed to participate in the research and fulfilled the selection criteria signed an institutionally-approved Informed Consent Form that was countersigned by the legal guardian if the subjects were under the age of 18 years. All testing was conducted according to the Statement on Human Experimentation (National Health and Medical Research Council, 1992).

\section{Participants}

Participants were recruited from regional netball teams. Netball is a ballsport that is typically played by females in the Commonwealth countries, and is similar to basketball. Sudden acceleration and deceleration movements, fast changes of direction, and catching a ball in the air followed by a landing task are typical features of the sport $[43,44]$. These movements place considerable loads on knee joint structures, and netball is readily acknowledged as a particularly high-risk sport for ACL injury [23]. Initial contact with the participants was made via the regional netball coordinator. A research associate then visited all teams during a preseason training session, and the research project was discussed with all athletes present. Eleven female netball players volunteered to participate in the study. The mean age was 16.3 years (SD 0.7, range 16-18) with a mean height of $164.0 \mathrm{~cm}$ (SD 6.2, range 154-172) and mean body mass of $60.7 \mathrm{~kg}$ (SD 6.3, range 47-72).

As netball is a sport played almost exclusively by females, it was not possible to recruit male netball players for a control group. The male control group instead consisted of an age- 
matched cohort selected from a local Rugby League Development Team. Although the physical demands of the two sports are clearly different, in the absence of elite competitive male netball players we believe age-matched male athletes from another sport are the most reasonable alternative control. Contact was made via the team doctor of the rugby football club. Similarly, the research associate presented the research project to all players during a training session. Six male subjects were included in the study. Their mean age was 16.0 years (SD 0), the mean height was $175 \mathrm{~cm}$ (SD 5.4, range 169-185) and a mean body mass of $75 \mathrm{~kg}$ (SD 16.4, range 58-105).

Female and male participants completed the IKDC questionnaire and were examined by an orthopaedic surgeon using the five tests described by Carter \& Wilkinson to rule out hyperlaxity [8]. Each female subject was also required to keep a diary documenting data on their menstrual cycle for three months prior, during, and three months following the test period. The following inclusion- and exclusion criteria were used for all female subjects:

\section{Inclusion Criteria}

1. Consistent menstrual cycles for at least three months, which included the period of time during the study

2. Menarche more than one year ago

3. No use of hormonal contraceptives or other hormones for three months prior to and during the test sessions

4. Normal range of motion of hip, knee and ankle joint

Subjects of the male group had to only meet criteria 4 in order to be included in the study.

\section{Exclusion Criteria:}

1. Hyperlaxity syndrome using the five tests as describe by Carter\&Wilkinson [8].

1. Any injury to the lower extremity twelve months prior or during the test period. 


\section{Outcome Measures}

Tibial acceleration profiles were investigated at each phase of the menstrual cycle: menstrual, follicular, ovulation and luteal. Male participants were tested twice, one week apart. This protocol for the male group was based on previous studies [4,10]. Beynnon et al. [4] and Deie et al. [10] could not demonstrate any changes in biomechanical nor hormonal variables during four consecutive tesing session for the male control group. As a result of these previous findings, the research group did not see the need for multiple repeated testing for the male cohort.

Venous blood samples were collected and analysed for luteinising hormone (LH), follicle stimulating hormone (FSH), estrogen and progesterone. The analysis allowed hormone levels to be matched with the specific menstrual phase of the testing date. Hormone analysis was performed at each of the testing sessions and hormone levels compared with Nakamura's data [36]. If hormone levels were inconsistent with levels reported by Nakamura [36], it was assumed that the test date was either miscalculated or that the cycle was anovulatory. In both cases, the subject was re-tested for that particular cycle. Using data from the menstrual-cycle diary, the average cycle for a particular female participant was calculated; the calculation of the testing sessions has been previously described [12].

\section{Tibial Acceleration-Time Measurements}

To measure tibial acceleration a uniaxial accelerometer was secured to the proximal proximal tibia tuberosity with both adhesive tape and Velcro straps. The accelerometer was mounted to a small aluminium plate that was moulded to fit the shape of the proximal tibia. A strap that ran through slots in the medial and lateral sides of the plate and attached at the back of the leg 
was used to further reinforce the plate/skin bond without causing discomfort [6]. The vertical axis of the accelerometer was aligned with the longitudinal axis of the tibia. Anteriorposterior accelerations were measured using the forward-backward axis of the accelerometer [9] (Figure 1). Tibial acceleration-time data were recorded and edited according to the methods used by Lafortune and Henning [27]. Peak tibial acceleration (PTA), time to peak tibial acceleration (TPTA), and time to zero tibial acceleration (TZTA) were recorded in gravities (g's) and the temporal values for each were measured in milliseconds.

In accordance with Steele $[43,44]$, female and male subjects performed a dynamic and abrupt deceleration task in which they accelerated forward towards an in-ground force plate (AMTI BP400800 - 2000, American Mechanical Technology Inc., Watertown, Massachusetts) installed in a flat cement floor to receive a chest level pass, landed on the test limb in singlelimb stance, and stabilized their position without raising the landing foot. This protocol not only served to replicate the in-play situation associated with netball, but also to deviate the subject's attention from concentrating on landing. Landing was deemed successful if the subject landed on the force plate while catching the ball during the flight phase with no subsequent hopping, extra steps or other uncontrolled landing motions (Figure 1). Three consecutive run-ups and jumps were recorded and averaged following a familiarization session of five attempts. Only landings with the dominant leg were analysed.

\section{Statistical Analysis}

The present study was designed to detect a $10 \%$ difference between testing sessions for tibial acceleration profiles with a mean standard deviation of $10 \%$ for the female group. The sample size calculation using standard assumptions of $\alpha=0.05$ and power of $80 \%$ indicated that nine subjects for the female study group group were needed. 
Hormone levels were presented in a descriptive fashion and compared to Nakamuras reference table [36]. A particular test session was included when hormone levels were within the $95 \%$ confidence interval as outlined by Nakamura [36]. If hormone values were not within the confidence interval as described by Nakamura, a two-tailed one sample Student's t-test was used to assess whether the level was significantly different from the reference value. If the alpha level was found to be less than 0.05 the result was excluded. Particpants were then retested during the next cycle. Mean and standard deviations of error were calculated for measures obtained at each testing session.

Repeated measures of ANOVA were used to assess tibial acceleration measures between testing sessions for the same individual. In the male group, the data from the two test sessions were compared using a paired-sample Student t-test. If the results were not significantly different, the mean of the two sessions was calculated and its value used to compare to the female group using ANOVA. Pearsons moment correlations were used to assess the relationships between estrogen- and, LH-levels, and tibial acceleration for all occasions. Statistical analysis was performed using Systat (version 13; Systat, Chicago, IL). 


\section{Results}

\section{Blood Analysis}

In the female study group, progesterone, estrogen, LH and FSH of all female subjects (Table 1) were found to be within Nakamuras reference values [36]. Significant differences $(P=0.0005-0.01)$ were observed in estrogen levels between ovulation, menstrual and follicular phase with the highest level observed during ovulation. In the male control group (Table 2) estrogen levels of were significantly $(P=0.0001)$ lower than in the female group at all times. There was no significant $(P=0.11)$ in-between test difference for hormone serum levels in the male cohort.

\section{Tibial Acceleration Profiles}

Female Study Group

Figure 2 shows the mean values for PTA, TPTA and TZTA. There was no significant difference between the cycles for PTA $(P=0.57)$. There were significant differences between menstruation and follicular $(P=0.04)$, menstruation and ovulation $(P=0.001)$, menstruation and luteal $(P=0.002)$ and follicular phase and ovulation for TPTA $(P=0.007)$. There was no significant between test session difference for TZTA $(P=0.59)$.

\section{Male Control Group}

Figure 3 demonstrates the mean values for TPA, TPTA and TZTA. There were no significant between test session differences for PTA $(P=0.48)$, TPTA $(P=0.29)$ and TZTA $(P=0.08)$. Subsequently the results for all variables were averaged and used for comparison with the female study group. The mean for PTA was $7.27 \mathrm{~g}$ (SD 2.34), for TPTA 27.25 ms (SD 8.06) and for TZTA was $48.38 \mathrm{~ms}$ (SD 8.06). 
Tibial acceleration profiles between groups and phases

Repeated measures of ANOVA revealed no significant between group differences for PTA $(P=0.1)$ and TZTA $(P=0.48)$ between the male control group and the female study group. However, significant between group differences were observed for TPTA $(P=0.001)$.

Tibial Relationships between estrogen- and LH-levels and tibial acceleration profiles for all phases and groups

For the female group a strong and significant negative relationship ( $r=-.075, p=0.03)$ was observed between estrogen-levels and PTA. For the male group this relationship was significant $(\mathrm{p}=0.01)$ but weak $(\mathrm{r}=-0.19)$. For the female group a strong and significant relationship ( $\mathrm{r}=0.58, \mathrm{p}=0.04)$ was observed between estrogen-levels and TPTA; for the male group these relationships were also significant $(\mathrm{p}=0.01)$; however, the correlation was only weak (r=0.16). Significant but weak relationships $(r=(-) 0.001-(-) 0.003 ; p=0.01-0.02)$ were observed between LH-levels and TZTA. For both the female and male group relationships between LH-levels and tibial acceleration profiles were non-significant and weak (Table 3). 


\section{Discussion}

This study investigated changes in the tibial acceleration profiles of young female athletes across their menstrual cycle. These measures provide a dynamic measure of tibial shock attenuation during a standardised landing task [33], and serve as an indirect indicator of dynamic knee stability $[6,27,28]$. The most important finding of the present study was that fluctuations of serum estrogen in young females have a significant effect on tibial acceleration profiles in young females during the menstrual cycle.

Peak tibial acceleration (PTA) serves as a descriptor for tibial shock attenuation and is an indirect measure of the ACL loading vector during initial ground contact [6,33]. In this study, significant within group differences in PTA were not observed between the different phases of the menstrual cycle $(P=0.57)$. The male group demonstrated overall higher but non-significant $(P=0.48)$ differences in PTA between independent testing sessions. The difference between the male and female groups was not significant $(P=0.21)$. Gender differences during this dynamic landing task may reflect an increased peak torque resulting from the larger cross-sectional muscle diameter and higher body mass typical of males $[15,29]$, and the characteristically lower peak torque values in women (1). To define PTA in

terms of gravities $(\mathrm{g})$, calculations must include the body mass $\left(\mathrm{g}=\mathrm{m}^{3} \mathrm{~kg}^{-1} \mathrm{~s}^{-2}\right)$, and given these caveats PTA is most likely not an important indicator of neuromuscular function. Albeit in different populations, Bryant et al. also reported that PTA was not related to knee function following ACL injury and ACL reconstruction [7].

Time to zero tibial acceleration (TZTA) is the duration of positive axial acceleration, and represents the time to attain constant velocity during deceleration [27,28,33]. A shorter 
duration TZTA, as a measure of greater dynamic stability, indicates better neuromuscular control $[6,33]$. In this study, the female group exhibited no significant difference between the different phases of the menstrual cycle for TZTA $(P=0.59)$. The male group demonstrated overall higher values, but non-significant differences between two independent sessions $(P=0.08)$ for TZTA. Again, the difference between the male and female groups was not significant $(P=0.48)$. Greater passive joint laxity in females is one plausible explanation for the differences we observed between the two groups. Shultz, et al. demonstrated that females had significantly $(P=0.023)$ greater knee laxity than males, and these gender differences varied by day of the menstrual cycle $(P=0.016)$ [41]. Earlier Rozzi, et al. measured knee joint laxity in both female and male soccer and basketball players, and demonstrated that women have significantly $(P=0.002)$ greater knee joint laxity [39]. In a prior publication investigating anterior knee laxity during the menstrual cycle, we could not demonstrate a significant effect $(P>0.05)[12]$.

In our 2007 study, anterior knee laxity increased non-significantly by an average of a $0.2 \mathrm{~mm}$ (3.9\%) from the onset of menstruation to the mid-follicular phase. At ovulation, anterior knee laxity increased non-significantly by a further $0.5 \mathrm{~mm}(10 \%)$ compared to the mid-follicular phase. Following ovulation, anterior knee laxity decreased non-significantly and was $0.5 \mathrm{~mm}$ $(10 \%)$ lower at the time of the luteal phase [12]. However, anterior knee laxity was significantly $(P=0.05)$ higher in the female group compared to the male control group. This increased knee laxity in women has almost certainly contributed to these findings, but perhaps these values do not fully reflect all potential neuromuscular responses to the changing hormonal environment [12]. 
Time to peak tibial acceleration (TPTA) is an important determinant of knee stability and functionality [6,28]; a short TPTA indicates good neuromuscular control with pre-activation of lower extremity muscles anticipating the impact prior to landing [6,33]. In this study, TPTA increased steadily throughout the menstrual cycle, reaching peak values in the female group at ovulation. Significant within group differences were observed between week one and two $(P=0.04)$, week one and three $(P=0.001)$, week one and four $(P=0.002)$, and week two and three $(P=0.007)$ of the menstrual cycle. The male group exhibited lower values, but non-significant differences between testing sessions $(P=0.29)$ for TPTA, while the difference between the male and female group was significant $(P=0.001)$. This clearly demonstrates male subjects had the ability to arrest tibial acceleration more quickly, and suggests they have better neuromuscular control. The longer TPTA in the female group implies neuromuscular control is less robust, while also an indication of inherently higher anterior tibial translation.

It may be argued that a $12 \mathrm{~ms}$ difference during the menstrual cycle is not of any clinical relevance. However, several researchers have reported on reflex response times for both the hamstring and quadriceps muscle groups in normal subjects compared to subjects with a variety of knee conditions $[2,34,46,49]$. Reflex responses in the normal group ranged from 22-40ms; given these figures the difference we report here of $12 \mathrm{~ms}$ therefore corresponds to a $30-54 \%$ delay. This is clearly a significant finding with the potential to contribute to athletic knee injuries.

Voight \& Wieder have demonstrated a significantly faster vastus lateralis response time in normal subjects compared to subjects with extensor mechanism dysfunction [49]. Witvrouw et al. investigated reflex response times of the vastus medialis obliquus and vastus lateralis in normal subjects and in subjects with patella-femoral pain syndrome [50]. Their study cohort 
showed a significantly lower ratio-reflex response time between the injured and uninured leg in the healthy control group. The authors concluded that patellofemoral pain is associated with a disturbed neuromotor control. Melnyk \& Gollhofer demonstrated that muscle fatigue impairs reflex response components by $35-40 \%$ and is associated with mechanical loss of knee stability [34]. As demonstrated previously by these other authors, relatively small changes in response time can be clinically relevant. It can therefore be reasonably assumed that the increase in TPTA we have observeded here is also significant, and should have similar implications with respect to knee stability. We believe the abnormal response time demonstrated in this study exposes the very genuine increased risk of injury to the anterior cruciate ligament during ovulation.

One can only speculate why these gender differences in the tibial acceleration profiles were observed. Gender differences in the tibial acceleration profiles could reflect differences in pre-activation of antagonist muscles, resulting in earlier eccentric contraction in the male athlete; several prior studies are consistent with this suggestion $[11,14,26,45]$. Gehring et al. assessed two-legged landings and noticed a significant delay $(P=0.05)$ in hamstring and quadriceps activation in females[14]. In addition, Sung \& Lee demonstrated significant time delays in muscle activation $(P=0.025)$ and lower EMG amplitudes when females descend stairs [45]. Furthermore, Ebben et al. investigated hamstring and quadriceps activation patterns using EMG and observed earlier activation of both muscle groups in males [11]. Finally, Krishnan et al. observed different between gender activation patterns for hamstring and quadriceps muscles; females displayed significantly lower $(P<0.001)$ muscle activity patterns, and also displayed a significantly higher magnitude of quadriceps $\quad(P<0.001)$ muscle activity than males to achieve the same relative force [26]. 
Gender specific knee kinematics may be partially responsible for the between gender differences in tibial acceleration profiles we observed. Malinzak et al. investigated dynamic sports activities and observed that women had less knee flexion, more knee valgus, greater quadriceps activation and lower hamstring activation [30]. Salci et al. (32) observed significantly lower knee and hip flexion angles in females during block landings [40]. Lephart et al. assessed gender differences in single-leg landing and forward hop tasks; with both activities, females had significantly less knee flexion, lower leg internal rotation, maximum angular displacement, and less knee flexion time to maximum angular displacement than males [29]. In the present study, we observed a negative and significant relationship ( $\mathrm{r}=-0.75, p=0.04)$ between PTA and estrogen levels, as well as TPTA $(\mathrm{r}=0.58$, $p=0.03$ ) and estrogen levels between testing sessions in the female group. Furthermore, when examining the different phases of the menstrual cycle and the male group and the different cycles within the female group, significant correlations ( $\mathrm{r}=0.6-0.99, p=0.016-0.04)$ were observed for all variables. Taken together, these results strongly suggest that estrogen fluctuations during a normal menstrual cycle have an influence on knee kinematics and kinetics in females.

To our knowledge, Clark et al. is the only prior study investigating tibial acceleration profile changes across the menstrual cycle [9]. They identified significant $(P=0.011)$ variability in the tibial acceleration profiles during treadmill running in young women at different stages of their menstrual cycle. Other researchers have investigated different measures of knee stability which indirectly support the findings of the present research $[10,22,41]$. For example, HicksLittle et al. reported a significant $(p=0.05)$ increase in anterior tibial displacement as an indirect indicator of shock attenuation between ovulation and other phases of the menstrual cycle [22]. Deie et al. studied knee laxity in 16 women across the menstrual cycle using a 
KT-2000 arthrometer, and demonstrated significant differences across the cycle [10]. Shultz et al. measured daily hormone levels and knee laxity during the menstrual cycle in 22 females, and reported knee laxity varied significantly $(p=0.016)$ by the day of the menstrual cycle [41].

The current project has limitations; it is difficult to recruit contemporary young women with a regular menstrual cycle who do not take oral contraceptive medication, and this could have resulted in selection bias. The ovulatory phase of the menstrual cycle lasts between two and four days; determination of the exact day of ovulation is difficult and cannot always be achieved. However, blood samples confirmed the estrogen and luteinizing hormone levels were significantly higher than at any of the other phases of the cycle. It can therefore be safely assumed that the likelihood of having committed an error in determining the ovulatory phase is small. The sample size calculation was based on a $10 \%$ difference with a standard deviation of $10 \%$ for all variables. While the differences in results between cycles for tibial acceleration profiles in the female group in the present study were above the calculated requirements, standard deviations were outside the used reference standard. This may have possibly caused type I errors; however, given the significant findings, it was felt that the chances of having committed a type I error were small. This is consistent with the results previously published in other studies using similar methods and sample sizes $[1,19,20]$.

Test-retest reliability has not been assessed in this project but has been reported by Turcot, et al. for 3-dimensional linear accelerations of the tibia and femur, and found to be 0.75 at both slow and fast speeds [47]. It could therefore be assumed the results for tibial acceleration profile measurements during the four testing sessions in the female group of this study were reliable. Finally, tibial acceleration profiles were measured using the skin-mounting 
technique previously validated by several authors $[28,29,31]$. In the present study, if the skin-mounted accelerometer moved during testing, or changed location between test sessions, intra-individual tibial acceleration profiles in the male group may have resulted in different values between test sessions.

In conclusion, these results strongly suggest serum estrogen fluctuations have a significant effect on tibial acceleration profiles in young females across the menstrual cycle. These results further suggest the female musculoskeletal system needs to constantly adjust to the changing hormonal environment, and adapt neuromuscular strategies to minimize the risk of injury. The constant need for neuromuscular adaptations may itself potentially expose the ACL to an increased risk of injury, and this may have further implications for the prevention ACL injuries in the female athlete. 


\section{References}

1. Abt JP, Sell TC, Laudner KG, McCrory JL, Loucks TL, Berga SL, Lephart SM (2007) Neuromuscular and biomechanical characteristics do not vary across the menstrual cycle. Knee Surg Sports Traumatol Arthrosc; 15:901-907

2. Bell DG, Jacobs I (1986) Electromechanical response times and rate of force development in males and females. Med Sci Sports Exerc, 18:31-36

3. Bell DR, Blackburn JT, Hackney AC, Marshall SW, Beutler AI, Padua DA (2014) Jumplanding biomechanics and knee-laxity change across the menstrual cycle in women with anterior cruciate ligament reconstruction. J Athl Train; 40 (2):154-162

4. Beynnon BD, Bernstein I, Belisle A ( 2005) The effect of estradiol and progesterone on knee and ankle laxity. Am J Sports Med, 33:1298-1304

5. Brophy RH, Silvers HJ, Mandelbaum BR (2010) Anterior cruciate ligament injuries: etiology and prevention. Sports Med Arthrosc Rev, 18:2-11

6 Bryant AL ( 2007) Knee function and neuromuscular adaptations following ACL rupture and reconstruction. PhD thesis, University of Wollongong

7. Bryant AL, Newton RU, Steele J ( 2009) Successful feed-forward strategies following ACL injury and reconstruction. J Electromyogr Kinesiol, 9:988-997

8. Carter C, Wilkinson J (1964): Persistent laxity and congenital dislocation of the hip. J. Bone Joint Br., 62:40-45

9. Clark RA, Bartold S, Bryant AL (2010) Tibial acceleration variability during consecutive gait cycles is influenced by the menstrual cycle. Clin Biomech, 25:557-562 
10. Deie M, Sakamaki Y, Sumen Y, Urabe Y, Ikuta Y (2002) Anterior knee laxity in young women varies with their cycle. Int Orthop; 26:154-156

11. Ebben WP, Fauth ML, Petushek E, Garceau LR, Hsu BE, Lutsch BN, Feldmann CR (2010) Gender-based analysis of hamstring and muscle activation during jump landings and cutting. J Strength Cond Res, 24:408-415

12. Eiling E., Bryant AL, Petersen W, Murphy A, Hohmann E (2007) Effects of menstrual cycle hormone fluctuations on muscultendinous stifness and knee joint laxity. Knee Surg Sport Traumatol Arthrosc, 15:126-132

13. Fagenbaum R., Darling WG (2003) Jump landing strategies in male and female College athletes and the implications of such strategies for anterior cruciate ligament injury.

Am J Sports Med, 31:233-40

14. Gehring D, Meinyk M, Gollhofer A (2009) Gender and fatigue have influence on knee joint control strategies during landing. Clin Biomech, 24:82-87

15. Griffin LY, Albohm MJ, Arendt EA, Bahr R, Beynnon BD, et al. (2006) Understanding and preventing noncontact anterior cruciate ligament injuries . A review of the Hunt Valley II Meeting, January 2005. Am J Sports Med, 34:1512-1532

16. Gwinn DE, Wilckens JH, McDevitt ER, Ross G, Kao TC (2000) The relative incidence of anterior cruciate ligament injury in men and women in the United States Naval Academy. Am J Sports Med, 27:98-102

17. Hakkinen K (1991) Force production characteristics of leg extensor, trunk flexor, and extensor muscles in male and female Basketball players. J Sports Med Phys Fitness, 31:325331

18. Hennig EM, LaFortune MA (1991) Relationships between ground reaction force and tibial bone acceleration parameters. Int J Sports Biomech, 7:303-309

19. Heitz NA, Eisenman PA, Beck CL, Walker JA (1999) Hormonal changes throughout the menstrual cycle and increased anterior cruciate ligament laxity in females. J Athletic Training 343:144-149

20. Hertel J, Williams NI, Olmsted-Kramer LC, Leidy HJ, Putkian M (2006) Neuromsucular performance and knee laxity do not change across the menstrual cylcle in female athletes. Knee Surg Sports Traumatol Arthrosc; 14:817-822

21. Hewett TE, Myer GD, Ford KR, Heidt RS Jr., Colosimo AJ, McLean SG, van den Bogert AJ, Paterno MV, Succop P (2005) Biomechanical measures of neuromuscular control and valgus loading of the knee predict anterior cruciate ligament injury risk in female athletes: a prospective study. Am J Sports Med, 33:492-501

22. Hicks-Little CA, Thatcher JR, Hauth J, Goldfuss AJ, Cordova ML (2007) Menstrual cycle stage and oral contraceptive effects on anterior tibial displacement in collegiate female athletes. J Sports Med Phys Fit; 45:255-260 
23. Hume PA, Steele JR (2000) A preliminary investigation of injury prevention strategies in Netball: are players heeding the advice? J Science Med Sport, 3:406-413

24. Ireland ML(2002) The female ACL: why is it more prone to injury? Orth Clin North Am; $33: 637-651$

25. Irmischer BS, Harris C, Pfeiffer RP, DeBeliso MA, Adams KJ, Shea KG (2004) Effects of a knee ligament injury prevention exercise program on impact forces in women. J Strength Cond Res; 18:703-7

26. Krishnan C, Huston K, Amendola A, Williams GN (2008) Quadriceps and hamstring muscle control in athletic males and females. J Orthop Res, 26:800-808

27. LaFortune MA, Hennig EM (1991) Contribution of angular motion and gravity to tibial accerelation. Med Science Sports Ex, 23:360-363

28. LaFortune MA, Hennig EM, Valiant GA (1995) Tibial shock measured with bone and skin mounted transducers. J Biomech, 28:989-993

29. Lephart SM, Ferris CM, Riemann BL, Myers JB, Fu FH (2002) Gender differences in strength and lower extremity kinematics during landing. Clin Orthop Rel Res, 401:162-169

30. Malinzak RA, Colby SM, Kirkendall DT, Yu B, Garrett WE (2001) A comparison of knee joint motion patterns between men and women in selected athletic tasks. Clin Biomech (Bristol, Avon), 16:438-445

31. Manal K, McClay Davis I, Galinat B, Stanhope S (2003) The accuracy of estimating proximal tibial translation during natural cadence walking: bone vs skin mounted targets. Clin Biomech, 18:126-131

32. McLean SG, Oh YK, Palmer ML, Lucey SM, Lucarelli DG, Ashton-Miller JA, Wojtys EM (2011) The relationship between tibial acceleration, tibial slope, and ACL strain during a simulated jump landing task. J Bone Joint Surg Am; 93 (14):1310-1317

33. Mc Nair PJ, Marshall RN (1994) Landing characteristics in subjects with normal and anterior cruciate ligament deficient knee joints. Arch Phys Med Rehabil, 75:584-589

34. Melnyk M, Gollhofer A (2007) Submaximal fatigue of the hamstrings impairs specific reflex components and knee stability. Knee Surg Sports Traumatol Arthrosc, 15:525-532

35. Myer GD, Ford KR, Hewett TE (2005) The effects of gender on quadriceps muscle activation strategies during a maneuver that mimics a high ACL injury risk position. J. Electromyogr Kinesiol, 5:181-189

36. Nakamura RM, Stanczyk FZ (1991) Immunoassays. In Mishell DR Jr, Davajan V, and Lobo RA, editors: Infertility, contraception and reproductive endocrinology, ed 3, 1991, Cambridge, Mass, Blackwell Scientific Publications. 
37. Prodromos CC, Han Y, Rogowski J, Joyce B, Shi K ( 2007) A meta-analysis of the incidence of anterior cruciate ligament tears as a function of gender, sport, and a knee injuryreduction regimen. Arthroscopy, 23:1320-1325

38. Romani W, Patrie J, Curl LA, Flaws JA (2003) The correlations between estradiol, estrone, estriol, progesterone and sex hormone-binding globulin and anterior cruciate ligament stiffness in healthy, active females. J Womens Health, 2:287-298

39. Rozzi SL, Lephart SM, Fu FH (1999) Effects of muscle fatigue on knee joint laxity and neuromuscular characteristics of male and female athletes. J Athlet Train, 34:106-114

40. Salci Y, Kentel BB, Heycan C, Akin S, Korkusuz F (2004) Comparison of landing maneuvers between male and female college Volleyball players. Clin Biomech (Bristol, Avon), 19:622-628

41. Shultz SJ, Sander TC, Kirk SE, Perrin DH (2005) Sex differences in knee joint laxity change across the female menstrual cycle. J. Sports Med. Phys. Fitness, 45:594-603

42. Shultz SJ, Schmitz RJ, Kong Y, Dudley WN, Beynnon BD, Nguyen AD, Kim H, Montgomery MM (2012) Cyclic variations in multiplanar knee laxity influence landing biomechanics. Med Sci Sports Exerc, 44 (5):900-909

43. Steele JR (1999) Biomechanical factors affecting performance in Netball. Implications for improving performance and injury reduction. Sports Med., 10:88-102

44. Steele JR, Brown JM (1999) Effects of chronic anterior cruciate ligament deficiency on muscle activation patterns during and abrupt deceleration task. Clin Biomech (Bristol, Avon), $14: 247-257$

45. Sung PS, Lee DC (2009) Gender differences in onset timing and activation of the muscles of the dominant knee during stair climbing. Knee, 16:375-380

46. Swanik CB, Lephart SM, Giraldo JL, DeMont RG, Fu FH (1999) Reactive mu scle firing of anterior cruciate ligament-injured females during functional activities. J Athl Training, 34:121-129

47. Turcot K, Aissaoui R, Boivin K, Hagenmeister N, Pelletier M, de Guise JA (2008) Testretest reliability and minimal change determination for 3-dimensional tibial and femoral accelerations during treadmill walking in knee osteoarthritis patients. Arch Phys Med Rehabil, 89:732-737

48. Viola RW, Steadman JR, Mair SD, Briggs KK, Sterett WI (1999) Anterior cruciate ligament injury incidence among male and female professional alpine skiers. Am J Sports Med; 27:792-5

49. Voight ML, Wieder DL (1991) Comparative reflex response times of vastus medialis oblique and vastus lateralis in normal subjects with extensor mechanism dysfunction. Am J Sports Med, 19:131-137 
50. Witvrouw E, Sneyers C, Lysens R, Victor J, Bellemans J (1996) Reflex response times of vastus medialis oblique and vastus lateralis in normal subjects and in subjects with patellofemoral pain syndrome. J Orthop Sports Phys Ther, 24 (3):160-165

51. Wojtys EM, Huston LJ, Boynton MD, Spindler KP, Lindenfeld TN (2002) The effect of the menstrual cycle on anterior cruciate ligament injuries in women as determined by hormone levels. Am J Sports Med, 30:182-188

52. Wojtys EM, Huston LJ, Schock HJ, Boylan JP, Ashton-Miller JA (2003) Gender differences in muscular protection of the knee in size matched athletes. J Bone Joint Surg Am; 85A:782-9

\section{Figure Legends}

\section{Figure 1:}

Dynamic landing of a subject on the designated area (force platform) in single limb stance with the dominant leg catching a netball during the flight phase.

\section{Figure 2:}

The figure demonstrates tibial acceleration profiles (PTA in g; TPTA and TZTA in ms) for the female group during the four phases of the menstrual cycle. PTA as a descriptor for tibial shock attenuation and indirect measure of the ACL loading during initial ground contact was lowest during menstruation and non-significantly higher during the other cycles. TZTA is the duration of positive axial acceleration and a shorter duration indicates greater dynamic stability and better neuromuscular control. Non-significant minor differences between the 
different phases of the menstrual cycle were observed. TPTA as an determinant of knee stability and functionality [indicating good neuromuscular control increased steadily throughout the menstrual cycle, reaching peak values in the female group at ovulation.

\section{Figure 3:}

The figure demonstrates tibial acceleration profiles (PTA in g; TPTA and TZTA in ms) for the male group during the two testing cycles. They demonstrated overall higher values for PTA and TZTA and, lower values for TPTA. The differences between testing sessions were not significant.

Table 1: Overview of hormone levels including standard deviations during the menstrual cycle of the female study group

\begin{tabular}{|c|c|c|c|c|}
\hline & Menstrual phase & Follicular phase & Ovulation & Luteal phase \\
\hline $\mathrm{LH}(\mathrm{IU} / \mathrm{l})$ & $3.1( \pm 1.65)$ & $6.64( \pm 3.7)$ & $22.09( \pm 20.9)$ & $6.18( \pm 4.7)$ \\
\hline $\mathrm{FSH}(\mathrm{IU} / \mathrm{l})$ & $5.05( \pm 1.85)$ & $6.66( \pm 1.7)$ & $7.78( \pm 4.9)$ & $3.71( \pm 2.0)$ \\
\hline Estrogen $(\mathrm{nmol} / \mathrm{l})$ & $105.41( \pm 36.9)$ & $175.36( \pm 50.5)$ & $510.4( \pm 290.4)$ & $336.26( \pm 168)$ \\
\hline Progesterone $(\mathrm{pmol} / \mathrm{l})$ & $0.66( \pm 0.29)$ & $0.48( \pm 0.2)$ & $6.06( \pm 1.1)$ & $20.66( \pm 2.2)$ \\
\hline
\end{tabular}


Table 2: Overview of hormone levels including standard deviations between test sessions for the male control group.

\begin{tabular}{|c|c|c|c|c|}
\hline & Test 1 & Test 2 & Mean & p-levels \\
\hline $\mathrm{LH}(\mathrm{IU} / \mathrm{l})$ & $6.06( \pm 0.7)$ & $6.32( \pm 0.7)$ & $6.19( \pm 0.7)$ & 0.47 \\
\hline $\mathrm{FSH}(\mathrm{IU} / \mathrm{l})$ & $3.95( \pm 2.5)$ & $3.77( \pm 2.2)$ & $3.86( \pm 2.3)$ & 0.45 \\
\hline Estrogen $(\mathrm{nmol} / \mathrm{l})$ & $83.4( \pm 9.3)$ & $58.8( \pm 23)$ & $71.1( \pm 16.1)$ & 0.11 \\
\hline Progesterone (pmol/l) & $0.37( \pm 0.2)$ & $0.14( \pm) .12)$ & $0.25( \pm 0.16)$ & 0.08 \\
\hline
\end{tabular}


Table 3: Tibial Relationships between estrogen- and LH-levels and tibial acceleration profiles for all phases and groups

\begin{tabular}{|c|c|c|c|c|}
\hline & female & $p$-levels & male & $p$-levels \\
\hline Estrogen-PTA & -0.75 & 0.03 & -0.19 & 0.01 \\
\hline Estrogen-TPTA & 0.58 & 0.04 & 0.16 & 0.01 \\
\hline Estrogen-TZTA & -0.01 & 0.0001 & -0.003 & 0.002 \\
\hline LH-PTA & 0.1 & 0.09 & 0.006 & 0.3 \\
\hline LH-TPTA & 0.22 & 0.29 & 0.26 & 0.62 \\
\hline LH-TZTA & 0.04 & 0.19 & -0.004 & 0.18 \\
\hline
\end{tabular}




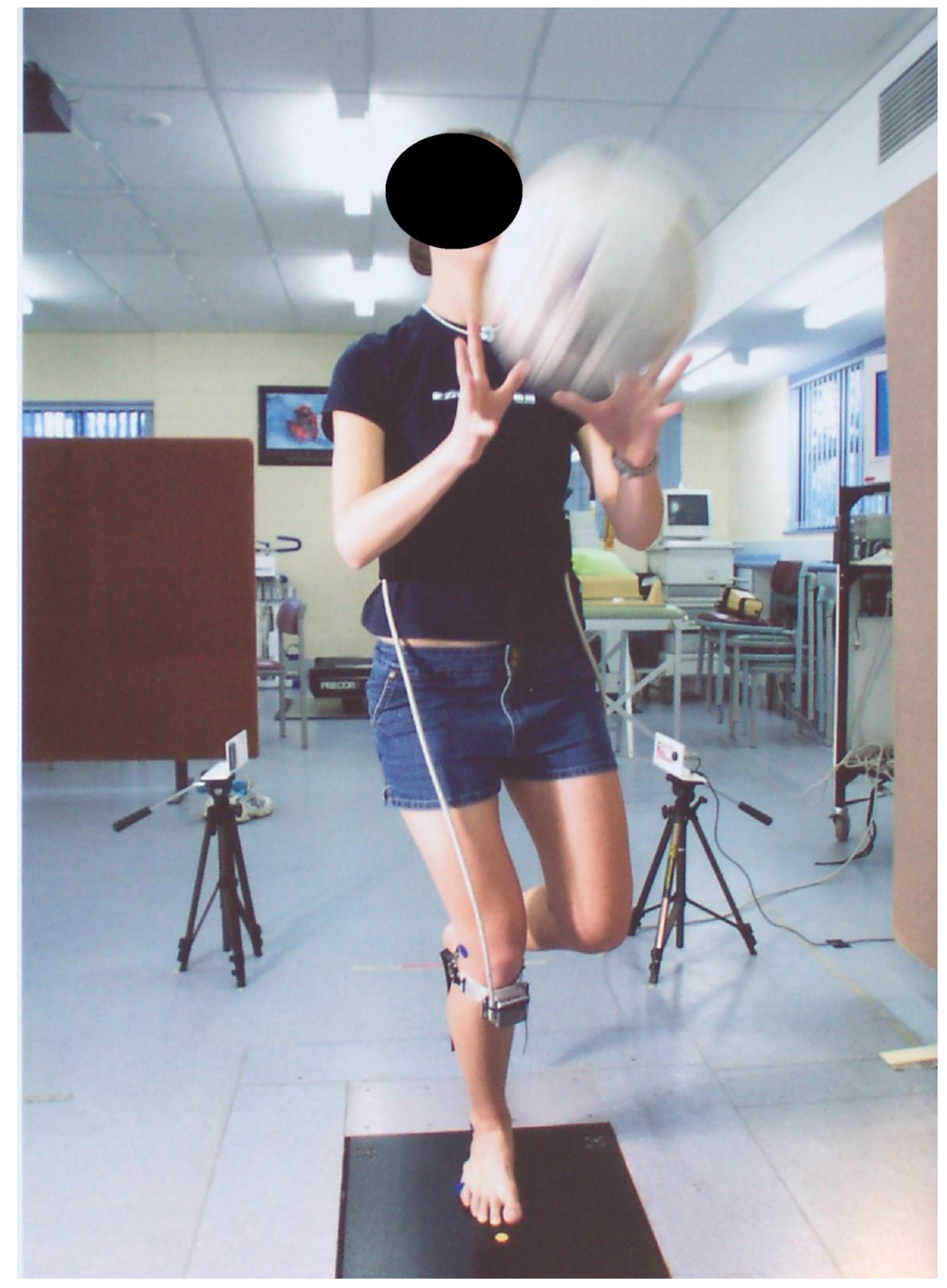


Tibial Acceleration Gradients

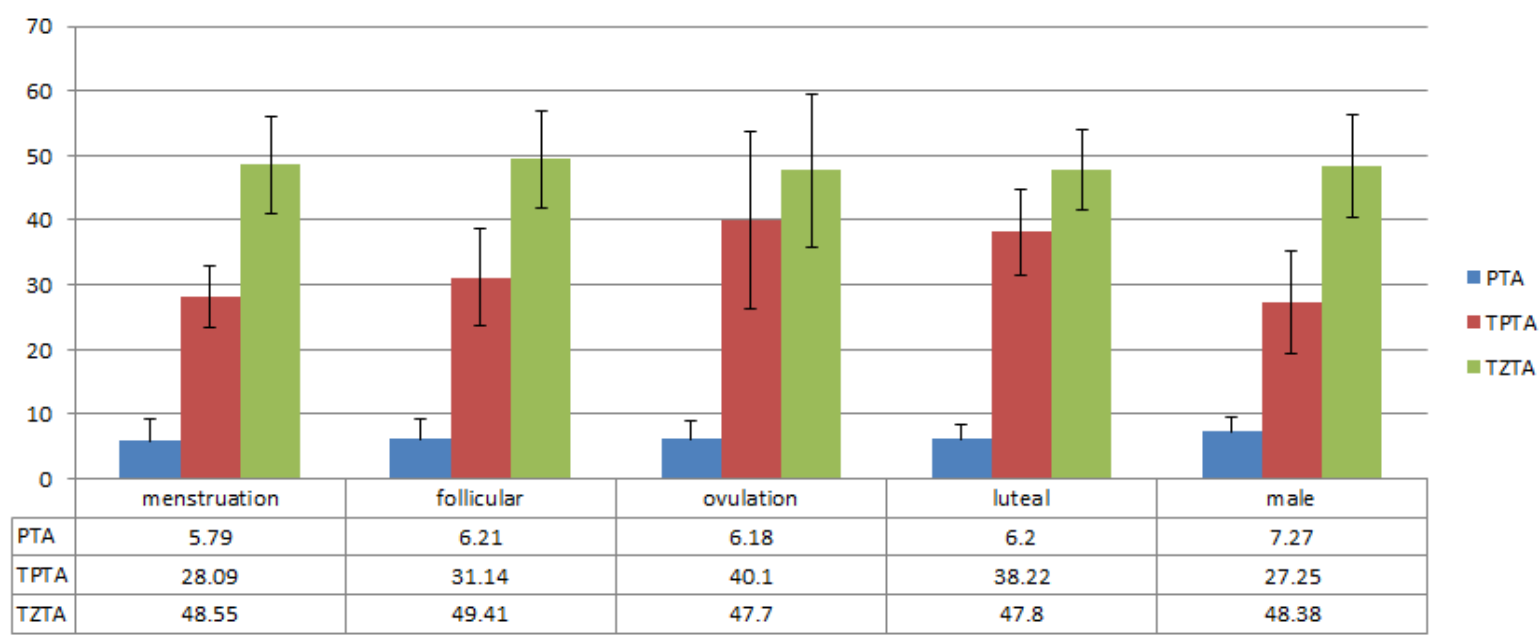

Tibial Acceleration Gradients For The Male Group

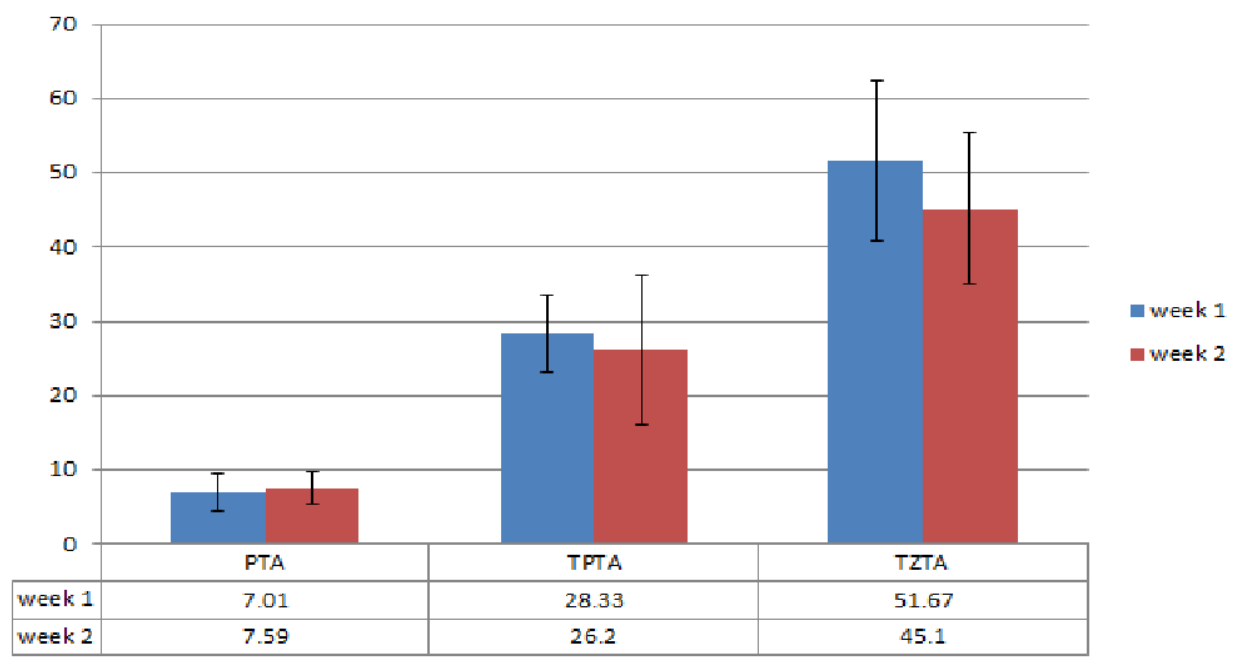

